\title{
FATORES DE RISCO PARA ALTERAÇÕES CITOPATOLÓGICAS CERVICAIS EM PACIENTES ATENDIDAS EM UNIDADE DE SAÚDE
}

\author{
RISK FACTORS FOR CERVICAL CYTOPATHOLOGICAL CHANGES IN \\ PATIENTS SERVED IN A HEALTH UNIT
}

\author{
Jolana Cristina Cavalheiri *1, Micheli Brum Alves ${ }^{1}$, Géssica Tuani Teixeira ${ }^{1}$, Marcela \\ Gonçalves Trevisan' ${ }^{1}$, Lediana Dalla Costa ${ }^{1}$, Alessandro Rodrigues Perondi ${ }^{1}$ \\ ${ }^{1}$ Universidade Paranaense, Francisco Beltrão, Paraná, Brasil. \\ *Autor Correspondente: Avenida Luiz Antonio Faedo, 1181, Ap. 403, Francisco Beltrão, Pr. \\ CEP:85601-275 E-mail: jolana@prof.unipar.br Telefone +55 (46) 99971-0091
}

\begin{abstract}
RESUMO
O objetivo deste estudo foi identificar os fatores de riscos associados às alterações citopatológicas cervicais em pacientes atendidas em uma unidade de saúde. Tratase de um estudo descritivo, de campo, documental, transversal, com abordagem quantitativa, realizado por meio da aplicação de questionário semiestruturado, avaliação de prontuários e mensuração de peso, estatura e cintura. A amostra constituiu-se de 70 mulheres em acompanhamento ginecológico por alteração de colo uterino. No presente estudo identificou-se a faixa etária com média de idade de 29,04 anos, representando risco de 2,53 as chances de desenvolver lesões intraepiteliais; de pele branca $(68,6 \%)$, refletindo em 7,12 as chances de evolução para lesões. A maioria das participantes $(41,4 \%)$ informou uma renda de até 2 salários mínimos, o que ofereceu um fator de risco de 1,39 chances para lesões intraepiteliais. O consumo de bebidas alcóolicas $(41,4 \%)$, uso de contraceptivo oral $(35,7 \%)$, histórico familiar de câncer uterino $(12,9 \%)$ e a exposição ao agrotóxico $(28,6 \%)$ constituem chance de 2,$49 ; 1,41 ; 4,51$ e 6,51 , respectivamente, para alteração intraepitelial. O perfil de mulheres com alteração citopatológica é de jovens, casadas, brancas e com baixa escolarização, as quais fazem uso de método contraceptivo do tipo oral e não utilizam o método de barreira. Afirmase, ainda, que este estudo possibilitará o desenvolvimento de pesquisas futuras.

Palavras-chaves: Fator de Risco; Câncer de colo uterino; Papanicolau; Saúde da mulher; Periodicidade.
\end{abstract}

\begin{abstract}
The objective of this study was to identify the risk factors associated to cervical cytopathologic changes in patients attended at a health unit. This is a descriptive, fieldbased, documentary, cross-sectional study with a quantitative approach, performed through the application of a semi-structured questionnaire, evaluation of medical records and measurement of weight, height and waist. The sample consisted in 70 women, in gynecological follow-up due to alteration of the uterine cervix. In the present study, a group with an age range of 29.04 years was identified, offering a 2.53 risk chances of developing intraepithelial lesions; with white skin (68.6\%), composing a 7.12 increase in chances of getting the injuries. The majority of the participants $(41.4 \%)$ reported an income of up to 2 minimum wages, which offered a risk factor of 1.39 of chances for intraepithelial lesions. The consumption of alcoholic beverages (41.4\%), use of oral contraceptives (35.7\%), family history of uterine cancer $(12,9 \%)$ and exposure to pesticides $(28.6 \%)$ offer odds of $1.41 ; 4,51$ and 2,49 , respectively, for intraepithelial alteration. The profile of women with cytopathological alteration is young, married, white and with low schooling, who use oral contraceptive methods and do not use the barrier method. It is also affirmed that this study will enable the development of future research.
\end{abstract}

Keywords: Risk Factor; Uterine cervix cancer; Papanicolau; Women's health; Frequency. 


\section{EM UNIDADE DE SAÚDE}

\section{INTRODUÇÃO}

$\mathrm{Na}$ atualidade, constata-se que o câncer de colo do útero é considerado um importante problema de saúde pública no Brasil, constituindo o terceiro tipo de câncer que mais acomete a população feminina, apresentando um índice de aproximadamente $270 \mathrm{mil}$ óbitos por ano, no mundo. Essa neoplasia é rara em mulheres com idade inferior a 30 anos, aumentando progressivamente a morbimortalidade por volta da quarta e quinta décadas de vida (NASCIMENTO et al., 2015).

No Brasil, estimaram-se 16.370 novos casos de câncer de colo uterino no ano de 2018, destacando-se as regiões Nordeste (6.030) e Sudeste (4.420) como as mais incidentes, e a região Centro-oeste com o menor número de casos (1.490). Só no estado do Paraná são esperados 820 novos casos do câncer, compondo um risco de 14,15 casos a cada 100 mil mulheres (INCA, 2018).

Dentre as alterações citopatológicas detectadas com o exame de rastreamento, o Papanicolau, tem-se o processo inflamatório causado por agentes etiológicos, lesões pós-traumática, além de alterações precursoras do câncer de colo do útero (BEDIN; GASPARIN; PETILIN, 2017). Estas últimas são classificadas de acordo com a complexidade da lesão ou o grau de comprometimento do tecido lesionado: A lesão intraepitelial escamosa de baixo grau, conhecida pela antiga terminologia como neoplasia intraepitelial cervical 1 (NIC1) é de bom prognóstico e pode regredir espontaneamente, e a lesão intraepitelial escamosa de alto grau, que englobam o NIC 2, NIC 3 ou o câncer já instalado e é considerada de maior intensidade e pior prognóstico (MACHADO; SOUZA; GOLÇALVES, 2017).

Conforme o preconizado pelo Ministério da Saúde, deverá ser realizado o exame de detecção precoce em todas as mulheres sexualmente ativas, com idades entre 25 e 64 anos e com uma periodicidade anual. Após dois exames de resultados normais, passa a ser preconizado a cada três anos. Apesar da disponibilidade do exame nas unidades básicas de saúde de todo o país, é notável a baixa adesão das mulheres com menores condições socioeconômicas, principalmente nos estados mais pobres, o que dificulta a efetividade no rastreamento (BARCELOS et al., 2017).
Também, dentre as principais infecções sexualmente transmissíveis, o Papilomavírus Humano (HPV) é responsável por pelo menos $70 \%$ dos casos de câncer de colo uterino. Existem vários genótipos diferentes relacionados à infecção e que acometem mulheres em idade reprodutiva, destacando-se os subtipos 16 e 18 como os de maior grau oncogênico e que prevalecem na maioria dos casos do câncer cervical (DALLA LIBERA et al., 2016a).

Além do HPV, existem outros fatores que possuem relação com o desenvolvimento de lesões precursoras do câncer como as baixas condições socioeconômicas e de escolaridade, multipariedade, menarca e sexarca precoces, multiplicidade de parceiros sexuais e o histórico anterior de infecções genitais. Vale ressaltar, ainda, a não adesão aos métodos de barreira, o uso por tempo prolongado do tabaco e dos anticoncepcionais orais, além da baixa adesão ao exame preventivo por motivos de vergonha, tabus e dificuldade de acesso ao serviço de saúde (LOBO; ALMEIDA; OLIVEIRA, 2018).

Outros fatores como a obesidade, sedentarismo, maus hábitos alimentares, exposição a alguns fatores ambientais específicos e substâncias com alto grau oncogênico (como derivados do carvão e os agrotóxicos utilizados nas lavouras) também elevam as chances do desenvolvimento de neoplasias e são muito destacadas em estudos e pesquisas científicas (MUNHOZ et al., 2016).

Diante do exposto, este estudo objetivou identificar os fatores de riscos associados às alterações citopatológicas cervicais em pacientes atendidas em uma unidade de saúde.

\section{MATERIAL E MÉTODO}

Trata-se de um estudo descritivo, de campo, documental, transversal e de abordagem quantitativa, realizado em uma unidade de saúde de um município do Sudoeste do Paraná, que abrange um território de $735.111 \mathrm{~km}^{2}$, com uma população de aproximadamente de 88.465 pessoas (IBGE, 2017).

O ambiente de estudo foi uma unidade de saúde, que funciona como um centro de referência em ginecologia e dispõe de tratamentos relacionados a alterações diagnosticadas nos exames preventivos de mama e colo de útero (FRANCISCO BELTRÃO, 2018). 
Para a realização do estudo convidaram-se a participar todas as mulheres que constituíam os casos de acompanhamento na unidade de saúde com alguma alteração citopatológica cervical, as quais aceitaram participar da pesquisa, mediante assinatura do Termo de Consentimento Livre e Esclarecido TCLE. Excluíram-se da pesquisa as demais pacientes atendidas no serviço.

A coleta de dados ocorreu entre os meses de junho e agosto de 2018. Assim, as mulheres que aceitaram participar da pesquisa foram direcionadas a uma sala reservada para aplicação do questionário e mensuração de peso, estatura e cintura, constituindo a amostra 70 participantes.

Em relação às questões norteadoras do questionário, as mesmas abordaram o perfil geral das mulheres como idade, raça, cor, peso e estatura para averiguação do índice de massa corpórea (IMC), circunferência da cintura (CC), estado civil, escolaridade, número de filhos, idade da primeira gestação, renda familiar, hábitos de vida (tabagismo, etilismo, alimentação), prática de exercícios físicos, uso de anticoncepcional, de preservativos, periodicidade da realização do exame preventivo, o que as levava à não realização do mesmo, sexarca, menarca, número de parceiros sexuais nos últimos 12 meses, histórico ginecológico anterior de infecções genitais e infecções sexualmente transmissíveis (IST), o conhecimento sobre a vacina que protege contra o HPV e a realização da imunização, histórico familiar de câncer de colo do útero e exposição aos agrotóxicos.

Para mensuração da altura, a participante foi devidamente posicionada livre de adereços na cabeça, ereta, com os pés juntos e os braços estendidos ao longo do corpo, com a cabeça erguida, olhando para um ponto fixo na altura dos olhos, mantendo-a parada nesta posição, estando apoiada na parede/antropômetro. Da mesma forma, a mensuração do peso foi realizada com balança antropométrica, ligada anteriormente e a mensuração zerada, sendo a participante colocada sobre a balança com o mínimo de roupa, descalça, ereta com os pés juntos e os braços estendidos ao longo do corpo, conforme recomendações do Ministério da Saúde (BRASIL, 2004).

Já para a mensuração da circunferência da cintura, aferiu-se com a participante em pé, ereta, com o abdômen relaxado, na altura da cintura ou na menor curvatura localizada entre a costela e a crista ilíaca conforme preconizado pela Organização Internacional de Saúde (BRASIL, 2004).

A fim de não ocasionar prejuízo às participantes, a mensuração das medidas antropométricas e os questionários foram aplicados pela própria pesquisadora, mantendo o sigilo das integrantes. Ao final, também foram utilizados os prontuários das participantes para verificar o laudo do exame citopatológico, no qual foi coletado o resultado dos exames preventivos realizados.

Após a coleta dos dados, os mesmos foram submetidos à tabulação no Programa Excel e, posteriormente, no pacote estatístico Statistical Package for the Social Sciences - SPSS, versão 21.0.

A estatística descritiva foi utilizada para caracterização da amostra e distribuição das frequências das diferentes variáveis analisadas, calculando-se o Risco Relativo (ODDS RATTIO) para as variáveis idade entre 16 a 35 anos; cor (branca e parda); IMC (adequado e sobrepeso); estado civil (solteiras e casadas/união estável); escolaridade (até 9 anos de estudo e mais que 10 anos de estudo); renda familiar (menor que 2 salários mínimos e maior); consumo de bebida alcoólica (sim e não); fumo (sim e não); prática de exercício físico (sim e não); uso de anticoncepcional oral ( $\operatorname{sim}$ e não); uso de camisinha (sim e não); sexarca (menor que 15 anos e maior de 15 anos); número de parceiros sexuais (um parceiro ou mais de um parceiro); histórico familiar de câncer de colo de útero (sim e não); exposição a agrotóxicos (sim e não) e medida de circunferência da cintura (menor que $80 \mathrm{~cm}$ e maior ou igual a $80 \mathrm{~cm}$ ) com a variável lesões de baixo, alto grau e câncer de colo do útero (CCU).

Ademais, foram calculadas as medidas de dispersão e variabilidade como média, mediana, desvio padrão, máximo e mínima das variáveis de peso, estatura, cintura e IMC. Por meio dos dados coletados de altura e peso foi possível determinar o IMC de acordo com a equação de Ketelet ( $\mathrm{IMC}=\mathrm{kg} / \mathrm{m}^{2}$ ), que utiliza os pontos de corte estipulados pelo Ministério da saúde para adultos. Também foi considerada a CC aumentada através de pontos de corte recomendados, $\mathrm{CC} \geq 80 \mathrm{~cm}$ para mulheres (BRASIL, 2017).

Além disso, o estudo proporcionou confidencialidade e sigilo das informações sobre a participante, mantendo os aspectos éticos e legais mediante autorização do Comitê de ética em Pesquisa com Seres Humanos da Universidade Paranaense 
- UNIPAR, aprovado sob o Parecer $n^{\circ}$ 2.676.259, em 2018.

\section{RESULTADOS}

Dentre as 70 mulheres participantes do estudo, $50 \%$ possuíam idade entre 16 e 35 anos; $68,6 \%$ eram consideradas brancas; $45,7 \%$ casadas; $34,3 \%$ tinham Ensino Fundamental incompleto, 58,6\% tinham dois ou mais filhos e $41,4 \%$ possuíam renda familiar de dois salários mínimos, conforme tabela 1.

Já a tabela 2 apresenta as tendências de dispersão e variabilidade das variáveis. Quanto ao índice de massa corpórea, $35,7 \%$ das participantes classificaramse como adequadas, com IMC entre 18,5 e 24,9 , seguido de $32,9 \%$ com sobrepeso, variando de 25 a 29,9 , obesidade I com $20,0 \%$, classificando-as em 30 a 34,9 , obesidade II 5,7\%, entre 35 e 39,9. Somente 2,9\% apresentaram-se com obesidade III, compondo um IMC maior que 40. Ainda, classificaram-se com baixo peso $2,9 \%$ das mulheres, que apresentaram um índice menor de 18,5.

Quanto à medida da circunferência da cintura (CC), os dados apresentaram um número de $61,4 \%$ das mulheres com medida igual ou maior de $80 \mathrm{~cm}$, considerado um fator de risco para o desenvolvimento de doenças crônicas não transmissíveis e 38,6\% com medida menor que $80 \mathrm{~cm}$.
Tabela 1 - Características sociodemográficas de mulheres em acompanhamento por alteração citopatológica cervical - Francisco Beltrão, PR

\begin{tabular}{|c|c|c|}
\hline Variável & $\mathbf{N}$ & $\%$ \\
\hline \multicolumn{3}{|l|}{ Faixa de idade } \\
\hline De 16 a 35 anos & 35 & 50,0 \\
\hline De 36 a 50 anos & 19 & 27,1 \\
\hline De 51 a 65 anos & 14 & 20,0 \\
\hline$>65$ anos & 2 & 2,9 \\
\hline \multicolumn{3}{|l|}{ Raça/Cor } \\
\hline Branca & 48 & 68,6 \\
\hline Parda & 20 & 38,6 \\
\hline Negra & 1 & 1,4 \\
\hline Amarela & 1 & 1,4 \\
\hline \multicolumn{3}{|l|}{ Estado Civil } \\
\hline Casada & 32 & 45,7 \\
\hline Solteira & 20 & 28,6 \\
\hline União estável & 10 & 14,3 \\
\hline Separada & 6 & 8,6 \\
\hline Viúva & 2 & 2,9 \\
\hline \multicolumn{3}{|l|}{ Escolaridade } \\
\hline Ensino Fundamental incompleto & 24 & 34,3 \\
\hline Ensino Fundamental completo & 6 & 8,6 \\
\hline Ensino Médio incompleto & 7 & 10,0 \\
\hline Ensino Médio completo & 19 & 27,1 \\
\hline Ensino Superior incompleto & 5 & 7,1 \\
\hline Ensino Superior completo & 9 & 12,9 \\
\hline \multicolumn{3}{|l|}{ Número de filhos } \\
\hline Nenhum & 12 & 17,1 \\
\hline Apenas 1 & 14 & 24,3 \\
\hline Dois ou mais & 41 & 58,6 \\
\hline \multicolumn{3}{|l|}{ Renda familiar } \\
\hline 1 salário mínimo & 19 & 27,1 \\
\hline 2 salários mínimos & 29 & 41,4 \\
\hline 3 salários mínimos & 11 & 15,7 \\
\hline$>4$ salários mínimos & 10 & 14,3 \\
\hline Não respondeu & 1 & 1,4 \\
\hline
\end{tabular}

Fonte: Coleta de dados, 2018. 
Tabela 2 -Medidas de dispersão e variabilidade de peso, estatura, cintura e IMC de mulheres em acompanhamento por alteração citopatológica cervical - Francisco Beltrão, PR.

\begin{tabular}{l|l|l|l|l|l}
\hline Variáveis & Média & Mediana & $\begin{array}{l}\text { Desvio } \\
\text { Padrão }\end{array}$ & Mínima & Máxima \\
\hline Peso & 69,69 & 71,0 & 13,28 & 39,0 & 99,0 \\
\hline Estatura & 1,60 & 1,60 & 0,07 & 1,42 & 1,86 \\
\hline Cintura & 82,3 & 82,5 & 13,02 & 44,50 & 110,00 \\
\hline IMC & 27,02 & 26,35 & 5,48 & 16,20 & 44,50 \\
\hline
\end{tabular}

Fonte: Coleta de dados, 2018.

Quanto aos fatores de risco, 78,6\% das mulheres relataram não ser tabagistas, $18,6 \%$ fumavam diariamente e $2,9 \%$ comentaram ter o hábito de fumar, mas não diariamente. Das 21,4\% mulheres que admitiam o uso do tabaco, $7,1 \%$ consumiam cerca de 10 a 14 cigarros por dia, $5,7 \%$ de 20 a 29 cigarros, $2,9 \%$ de 1 a 4 cigarros e outras $2,9 \%$ de 30 a 39 cigarros/ dia. Quando questionado às que relataram não ser fumantes se, no passado, já fumaram, 49,9\% relataram que nunca fumaram, $18,6 \% \mathrm{sim}$, diariamente e $10 \%$ sim, esporadicamente.

Em relação ao consumo de bebidas alcoólicas, $41,4 \%$ das participantes confirmaram. Das que admitiram o consumo do álcool, 21,4\% relataram o hábito de beber em apenas 1 a 2 dias na semana. Somente $1,4 \%$ relatou o consumo diário.

Quanto ao consumo de alimentos como a carne vermelha, $34,3 \%$ referiram consumir com uma frequência de 3 a 4 dias por semana, 24,3\% afirmaram consumi-la diariamente e $7,1 \%$ raramente ingeriam esse tipo de carne. O consumo de doces como chocolates, bolos, biscoitos, balas e sorvetes foi referido por $31,4 \%$ das mulheres com uma frequência de 1 a 2 dias na semana, e $11,4 \%$, diariamente.

A ingestão de refrigerantes ou sucos artificiais foi relatada como quase nunca por $31,4 \%$ das mulheres. Quando questionado às participantes com que frequência trocavam uma refeição para comerem um lanche, 40,0\% afirmaram quase nunca e $35,7 \%$ de 1 a 2 dias na semana, conforme tabela 3.

O consumo de verduras e legumes foi destacado por $45,7 \%$ das mulheres em todos os dias da semana e o consumo de frutas por $50,0 \%$ todos os dias. A prática de exercícios físicos foi relatada por $54,3 \%$ das mulheres, das quais $31,4 \%$ destacaram a caminhada como a principal atividade, conforme tabela 4 .
Tabela 3 -Relação dos hábitos de vida e frequência do consumo de alimentos considerados não saudáveis, relatados por mulheres em acompanhamento por alteração citopatológica cervical - Francisco Beltrão, PR

\begin{tabular}{|c|c|c|}
\hline Variáveis & $\mathbf{N}$ & $\%$ \\
\hline \multicolumn{3}{|l|}{ Consumo de cigarros } \\
\hline Sim, diariamente & 13 & 18,6 \\
\hline Sim, mas não diariamente & 2 & 2,9 \\
\hline Não & 55 & 78,615 \\
\hline \multicolumn{3}{|l|}{$\begin{array}{l}\text { Frequência do consumo de bebida } \\
\text { alcoólica }\end{array}$} \\
\hline 1 a 2 dias por semana & 15 & 21,4 \\
\hline 3 a 4 dias por semana & 1 & 1,4 \\
\hline 5 a 6 dias por semana & 1 & 1,4 \\
\hline $\begin{array}{l}\text { Todos os dias (incluindo os sábados e } \\
\text { domingo) }\end{array}$ & 1 & 1,4 \\
\hline Menos de um dia por semana & 5 & 7,1 \\
\hline Menos de um dia por mês & 6 & 8,6 \\
\hline \multicolumn{3}{|l|}{$\begin{array}{l}\text { Frequência do consumo de carne } \\
\text { vermelha }\end{array}$} \\
\hline 1 a 2 dias por semana & 21 & 30,0 \\
\hline 3 a 4 dias por semana & 24 & 34,4 \\
\hline 5 a 6 dias por semana & 3 & 4,3 \\
\hline Todos os dias & 17 & 24,3 \\
\hline Quase nunca & 5 & 7,1 \\
\hline \multicolumn{3}{|l|}{ Refrigerantes ou sucos artificiais } \\
\hline 1 a 2 dias por semana & 18 & 25,7 \\
\hline 3 a 4 dias por semana & 6 & 8,6 \\
\hline 5 a 6 dias por semana & 3 & 4,3 \\
\hline Todos os dias & 9 & 12,9 \\
\hline Quase nunca & 22 & 31,4 \\
\hline Nunca & 12 & 17,1 \\
\hline \multicolumn{3}{|l|}{ Doces } \\
\hline 1 a 2 dias por semana & 22 & 31,4 \\
\hline 3 a 4 dias por semana & 14 & 20,0 \\
\hline 5 a 6 dias por semana & 4 & 5,7 \\
\hline Todos os dias & 8 & 11,4 \\
\hline Quase nunca & 17 & 24,3 \\
\hline Nunca & 5 & 7,1 \\
\hline \multicolumn{3}{|l|}{ Troca uma refeição por lanche } \\
\hline 1 a 2 dias por semana & 25 & 35,7 \\
\hline 3 a 4 dias por semana & 3 & 4,3 \\
\hline 5 a 6 dias por semana & 1 & 1,4 \\
\hline Quase nunca & 28 & 40,0 \\
\hline Nunca & 13 & 18,6 \\
\hline
\end{tabular}

Fonte: Coleta de dados, 2018. 
Quando perguntou-se às mulheres como classificavam o seu estado de saúde atual, 52,9\% consideravam-no como um bom estado de saúde, $34,3 \%$ como regular, $8,6 \%$ muito bom, $2,9 \%$ ruim e $1,4 \%$ não respondeu.

Tabela 4 -Fatores de proteção relatados pelas mulheres em acompanhamento por alteração citopatológica cervical, relacionados ao consumo de alimentos saudáveis e à prática de atividade física Francisco Beltrão, PR

\begin{tabular}{l|l|l}
\hline Variáveis & N & \% \\
\hline $\begin{array}{l}\text { Frequência do consumo de verduras e } \\
\text { legumes }\end{array}$ & & \\
\hline 1 a 2 dias por semana & 15 & 21,4 \\
\hline 3 a 4 dias por semana & 12 & 17,1 \\
\hline 5 a 6 dias por semana & 6 & 8,6 \\
\hline Todos os dias & 32 & 45,7 \\
\hline Quase nunca & 4 & 5,7 \\
\hline Nunca & 1 & 1,4 \\
\hline Frutas & & \\
\hline 1 a 2 dias por semana & 13 & 18,6 \\
\hline 3 a 4 dias por semana & 6 & 8,6 \\
\hline 5 a 6 dias por semana & 5 & 7,1 \\
\hline Todos os dias & 35 & 50,0 \\
\hline Quase nunca & 10 & 14,3 \\
\hline Não respondeu & 1 & 1,4 \\
\hline Tipo de atividade física & & \\
\hline Caminhada & 22 & 31,4 \\
\hline Academia & 7 & 10,0 \\
\hline Outro & 6 & 8,6 \\
\hline Caminhada + academia & 2 & 2,9 \\
\hline Caminhada + ciclismo & 1 & 1,4 \\
\hline Não pratica & 32 & 45,7 \\
\hline
\end{tabular}

Fonte: Coleta de dados, 2018.

A tabela 5, na sequência, demonstra os dados ginecológicos das mulheres em acompanhamento por alteração de colo uterino, no qual 51,4\% não faziam uso de método contraceptivo, $35,7 \%$ utilizavam o anticoncepcional oral, 30,0\% informaram não utilizar o método de barreira durante as relações e $57,1 \%$ começaram a ter relações sexuais com idade entre $15 \mathrm{e}$ 19 anos. A primeira menstruação foi relatada por $54,3 \%$ das mulheres entre os 12 e 15 anos e $68,6 \%$ tiveram, nos últimos 12 meses, apenas 1 parceiro sexual.
Tabela 5 -Dados ginecológicos de mulheres em acompanhamento por alteração citopatológica cervical - Francisco Beltrão, PR

\begin{tabular}{|c|c|c|}
\hline Variáveis & $\mathbf{N}$ & $\%$ \\
\hline \multicolumn{3}{|l|}{ Uso de contraceptivo } \\
\hline Anticoncepcional oral & 25 & 35,7 \\
\hline Injeções Hormonais & 7 & 10,0 \\
\hline Outro & 2 & 2,9 \\
\hline Não faz uso & 36 & 51,4 \\
\hline \multicolumn{3}{|l|}{ Uso de camisinha } \\
\hline Sempre & 19 & 27,1 \\
\hline Nunca & 21 & 30,0 \\
\hline Às vezes & 15 & 21,4 \\
\hline Não respondeu & 15 & 21,4 \\
\hline \multicolumn{3}{|c|}{$\begin{array}{l}\text { Frequência que realiza o exame } \\
\text { citopatológico }\end{array}$} \\
\hline De 3 em 3 meses & 4 & 5,7 \\
\hline De 6 em 6 meses & 12 & 17,1 \\
\hline Anualmente & 43 & 61,4 \\
\hline Raramente & 11 & 15,7 \\
\hline \multicolumn{3}{|l|}{ Sexarca } \\
\hline Entre 12 e 15 anos & 19 & 27,1 \\
\hline Entre 16 e 19 anos & 40 & 57,1 \\
\hline Acima de 19 anos & 9 & 12,9 \\
\hline Não respondeu & 2 & 2,9 \\
\hline \multicolumn{3}{|l|}{ Menarca } \\
\hline Entre 9 e 12 anos & 29 & 41,4 \\
\hline Entre 13 e 15 anos & 38 & 54,3 \\
\hline Acima de 15 anos & 2 & 2,9 \\
\hline Não lembra/ não respondeu & 1 & 1,4 \\
\hline \multicolumn{3}{|c|}{ Número de parceiros sexuais no último ano } \\
\hline 1 único parceiro & 48 & 68,6 \\
\hline 2 parceiros & 7 & 10,0 \\
\hline 3 parceiros & 2 & 2,9 \\
\hline 4 ou mais parceiros & 2 & 2,9 \\
\hline Nenhum & 8 & 11,4 \\
\hline Não respondeu & 3 & 4,3 \\
\hline \multicolumn{3}{|c|}{ Histórico de infecções genitais e IST } \\
\hline Candidíase & 14 & 20,0 \\
\hline Vaginose & 2 & 2,9 \\
\hline HPV & 16 & 22,9 \\
\hline Nenhum & 34 & 48,6 \\
\hline Candidíase $+\mathrm{HPV}$ & 4 & 5,7 \\
\hline \multicolumn{3}{|c|}{$\begin{array}{l}\text { Conhecimento sobre a vacina contra o } \\
\text { HPV }\end{array}$} \\
\hline Sim & 61 & 87,1 \\
\hline Não & 9 & 12,9 \\
\hline
\end{tabular}

Fonte: Coleta de dados, 2018. 
Ainda na tabela 5, 48,6\% das participantes não informaram nenhuma infecção genital ou infecção sexualmente transmissível, enquanto $22,9 \%$ tiveram contato com o Papilomavírus Humano. Dentre as pesquisadas, $87,1 \%$ informaram ter conhecimento sobre a vacina contra o HPV e as outras $12,9 \%$ nunca ouviram falar. Quando questionadas se haviam realizado a vacina, $7,1 \%$ responderam que receberam todas as doses, $2,9 \%$ que o esquema está incompleto e $90,0 \%$ não realizaram a mesma.

Quanto à realização do exame citopatológico, $61,4 \%$ efetivaram o exame anualmente e $15,7 \%$ raramente e, dentre estas últimas, 5,7\% não realizavam por falta de tempo, $4,3 \%$ por vergonha, $1,4 \%$ por não estar vivendo com um companheiro, por dificuldade no acesso do serviço de saúde, por não apresentar leucorréia e por motivo não informado $(1,4 \%)$.

Entre as 70 mulheres que aceitaram participar do estudo, $87,1 \%$ negaram histórico familiar de câncer de colo do útero, 71,4\% declararam nunca ter tido exposição a agrotóxicos e $28,6 \%$ já estiveram expostas ao agrofármaco em algum momento da vida. Dentre as mulheres que relataram a exposição, 12,9\% lavavam as roupas utilizadas durante a aplicação do agrotóxico, $8,6 \%$ tiveram contato durante a aplicação manual nas lavouras, 2,9\% lavavam as embalagens, realizavam a aplicação manual e, após, lavavam as roupas utilizadas e 1,4\% não informou o tipo de exposição.

Além dos dados supramencionados, o estudo proporcionou também identificar os diagnósticos habituais nas mulheres em acompanhamento ginecológico, sendo que $65,6 \%$ apresentaram-se com lesões de baixo grau e 34,3\% com lesões de alto grau, incluindo um caso $(1,4 \%)$ de câncer de colo do útero já instalado.

A tabela abaixo demonstra os fatores de risco para o desenvolvimento de lesões intraepiteliais de baixo e alto grau/CA. A idade 16 a 35 anos apresentou um aumento em 2,5 chances de desenvolver lesões de baixo e alto grau. A variável cor branca aumenta as chances em 7,1, enquanto o estado civil solteira, aumenta em 2,4. A renda familiar entre 1 e 2 salários mínimos aumenta o risco de 1,3 , o consumo de bebidas alcoólicas 2,4 chances e o uso de anticoncepcional oral 1,4 chances. Além disso, o histórico de câncer de colo do útero apresentou um importante fator de risco, com 4,5 chances a mais, assim como a exposição a agrotóxicos, com 6,5.
Tabela 6 -Fatores de risco para lesões de baixo e alto grau/CA em mulheres em acompanhamento citopatológico cervical - Francisco Beltrão, PR

\begin{tabular}{l|l|l}
\hline Fator de Risco & Valor & $\begin{array}{l}\text { Intervalo de } \\
\text { Confiança }\end{array}$ \\
\hline Lesões de baixo/ alto grau/CCU & & \\
\hline Idade entre 16 e 35 anos & 2,53 & $0,899-7,124$ \\
\hline Cor branca & 7,12 & $0,478-4,066$ \\
\hline Solteira & 2,49 & $0,836-7,438$ \\
\hline $\begin{array}{l}\text { Renda familiar de 1 a 2 salários } \\
\text { mínimos }\end{array}$ & 1,39 & $0,478-4,066$ \\
\hline Consumo de bebidas alcoólicas & 2,49 & $0,836-7,438$ \\
\hline Prática de exercício físico & 2,50 & $0,901-6,973$ \\
\hline Uso de anticoncepcional oral & 1,41 & $0,489-4,116$ \\
\hline Uso de camisinha & 5,95 & $1,241-28,534$ \\
\hline Histórico de CCU & 4,51 & $0,529-38,492$ \\
\hline Exposição a agrotóxicos & 6,51 & $1,363-31,173$ \\
\hline Circunferência da cintura $<80 \mathrm{~cm}$ & 1,27 & $0,451-3,589$ \\
\hline
\end{tabular}

Fonte: Coleta de dados, 2018.

Na variável uso de preservativo, observou-se um aumento em 5,9 as chances de desenvolver lesões, a prática de exercícios físicos de 2,5 chances e a medida da circunferência da cintura $<80 \mathrm{~cm}$ aumenta em 1,2 as chances de desenvolver as lesões intrapiteliais ou o próprio câncer, sendo estes vieses de causalidade reversa, já que a maioria das mulheres era casada, não realizava exercício físico e possuía uma circunferência aumentada.

\section{DISCUSSÃO}

O câncer cervical é um importante problema de saúde pública no mundo, e é considerado um dos tipos de câncer invasor que mais acomete o sexo feminino, destacando sua incidência, principalmente, nos países subdesenvolvidos. No Brasil, destaca-se como a quarta causa de mortalidade em mulheres, cuja estimativa em 2013 foi cerca de 5.430 óbitos por esse tipo de neoplasia (MOREIRA, 2017). Nos anos de 2016 e 2017 foram esperados mais de 16 mil casos, destacando a neoplasia entre os quatro tipos de câncer mais incidentes no país (ROCHA; BAHIA; ROCHA, 2016).

O carcinoma invasor cervical apresenta uma evolução lenta e pode levar até duas décadas para se desenvolver e ocasionar prejuízos irreversíveis. Esse tipo de neoplasia se inicia através do aparecimento de lesões intraepiteliais, consideradas precursoras do 


\section{EM UNIDADE DE SAÚDE}

câncer de colo do útero. Estas são detectadas através do exame Papanicolau e apresentam quase $100 \%$ de associação com a infecção pelo HPV (MACHADO, SOUZA, GONÇALVES, 2017).

Além disso, as alterações citopatológicas podem ser multifatoriais, originando-se através de uma junção de inúmeros fatores como os hábitos de vida, fatores sociais, ambientais, genéticos, ginecológicos, a ingesta inadequada de alimentos ricos em gordura, o sedentarismo e o sobrepeso, além da exposição excessiva a agentes nocivos como o tabaco e agrotóxicos. Isso, além de fatores não modificáveis, como a idade e histórico familiar (TIECKER; BAIDEIRA; BERLEZI, 2016).

Através da efetivação do presente estudo, constatou-se que a faixa etária mais acometida foi a de mulheres de 16 a 35 anos (50\%), com uma média de idade de 29,04 anos. Tal comprovação corrobora o estudo de Machado e Pires (2017), realizado em Ribeirão Pires, São Paulo, no qual se obteve uma média de idade de 31,61 anos. Outro estudo semelhante, realizado no Rio Grande do Sul, por Nobre et al. (2014), com uma amostra de 48 mulheres, identificou que a faixa etária mais acometida por alterações cervicais foi de 21 a 40 anos $(52,1 \%)$.

A presente variável apresentou-se como um importante fator de risco $(2,53)$ para o desenvolvimento de lesões intraepiteliais de baixo e alto graus, o que ocorre pela exposição precoce à infecção pelo Papilomavírus Humano. Da mesma forma, quando detectada em mulheres mais velhas ou de meia idade, justifica-se pelo período prolongado de evolução da doença (DALLA LIBERA et al., 2016a).

Quanto à cor da pele, observou-se a predominância na cor branca $(68,6 \%)$. Tais dados foram ratificados no estudo de Dias et al. (2015) realizado em Serranópolis, Minas Gerais, no qual $31,2 \%$ eram brancas. Entretanto, em estudo de Ribeiro et al. (2015), no Piauí, com 699 prontuários, observouse maior incidência de mulheres não brancas $(82,1 \%)$. Etnicamente, a região do Sudoeste do Paraná foi colonizada por alemães e italianos, o que contribui para esses resultados.

Outro fator averiguado foi que a grande maioria das mulheres $(45,7 \%)$ afirmou ser casada, equivalendo à pesquisa de Mello, Galle e Prado (2017), realizada em São Paulo, que destacou 58\% das entrevistadas com o mesmo estado civil. Concorda-se, aqui, com o estudo de Moraes et al. (2016), desenvolvido no interior do Estado de São Paulo, o qual corroborou que $47,5 \%$ das mulheres eram casadas. Entretanto, neste estudo, o estado civil solteira apresentou-se como fator de risco $(2,49)$, uma vez que se pressupõe que estas relacionam-se com mais de um parceiro, apresentando maior exposição ao HPV e infecções (ALMEIDA et al., 2015).

No que tange à escolaridade menor que 9 anos de estudo foi confirmada em $34,3 \%$ das pesquisadas, dados semelhantes citados por Dias et al. (2015), com 45,45\% das mulheres com Ensino Fundamental incompleto. Tal variável corrobora o estudo de Figueiredo et al. (2015) realizado em Januária, MG, no qual 38,7\% também apresentaram o mesmo grau de escolaridade. Supõe-se que a menor escolaridade interfira na compreensão das mulheres quanto à importância da realização do exame citopatológico, assim como no entendimento das práticas preventivas em saúde e do seguimento do tratamento correto.

Em relação ao número de filhos, 58,6\% afirmaram a multiparidade. Seguindo essa perspectiva, o estudo de Dias et al. (2015) demonstrou que 47,73\% também relataram ter de 3 a 5 filhos, corroborando, ainda, o estudo de Machado e Pires (2017), no qual 51,02\% das participantes informaram a mesma condição.

No estudo em apresentação, 41,4\% informaram uma renda de 2 salários mínimos, sendo este fator de risco $(1,39)$ para desenvolver lesões intraepiteliais. Dados estes foram ratificados por Figueiredo et al. (2015), no qual 59,1\% das mulheres apresentavam a mesma faixa de renda salarial. Inclusive, pesquisa realizada em Montes Claros, MG, por Silva, Coelho e Athayde (2016), com uma amostra de 198 mulheres, $55,6 \%$ também relataram uma renda familiar de 1 a 2 salários mínimos. A baixa renda familiar se relaciona à escassez de informação e baixa escolaridade, e, especialmente, nas alterações citopatológicas cervicais, que levam as mulheres a não realizarem o exame de detecção precoce, permitindo a progressão de lesões, as quais podem desencadear, mais tardiamente, o câncer de colo de útero (DALLA LIBERA et al., 2016b).

No que diz respeito ao índice de massa corpórea das participantes, 35,7\% apresentaram-se adequadas, seguido de sobrepeso com 32,9\%. A medida da circunferência da cintura demonstrou valores iguais ou maior que $80 \mathrm{~cm}$. Porém, observou-se um viés de causalidade reversa no quesito fator de risco, uma vez 
que o mesmo destacou significância $(1,27)$ na medida da cintura $<80 \mathrm{~cm}$. Segundo o estudo de Oliveira et al. (2014), realizado em Teresina, Piauí, com 51 mulheres de idades entre 25 e 64 anos, 41,2\% apresentaramse em sobrepeso e com uma circunferência acima do preconizado pelo Ministério da Saúde $(31,4 \%)$.

Afirma-se que os altos índices de sobrepeso e obesidade têm sido considerados um importante problema de saúde, tanto em países desenvolvidos como em desenvolvimento. Isto, porque essa condição tem acometido desde a população infantil até a adulta. Além do mais, o acúmulo de tecido adiposo corporal pode ocasionar inúmeras doenças crônicas, condicionando alterações significativas nos sistemas endócrino e metabólico do indivíduo, corroborando que em cerca de $42 \%$ há o desenvolvimento de certas neoplasias, como o câncer de colo do útero (CORDEIRO; FREITAS, 2016). Em estudo internacional realizado por Duoung et al. (2017), observou-se, também, a associação entre os altos índices de tecido adiposo com células cancerígenas, uma vez que os adipócitos podem levar a alterações fenotípicas nas células tumorais, influenciando diretamente na sua evolução.

Considera-se, ainda, o tabagismo, que foi relatado por $21,5 \%$ das mulheres, destacando um consumo de 10 a 14 cigarros por dia $(7,1 \%)$. Segundo Silva et al. (2015), o consumo de pelo menos 100 cigarros durante toda a trajetória da vida, já é o suficiente para desenvolver persistência viral, como ao Papilomavírus humano e ocasionar danos genômicos às células.

Já em estudo realizado por Bazzo et al. (2014), com 67 mulheres, no qual foram associados o hábito de fumar ao resultado dos exames cito e anatomopatológico, pôde-se observar que 83,6\% das voluntárias se diziam não fumantes e exibiram resultados citopatológicos negativos para alterações de colo do útero. Já as que informaram o hábito de fumar $(16,4 \%)$ tiveram os mesmos resultados, não apresentando agregações quando o hábito de fumar e o desenvolvimento de lesões citopatológicas.

A respeito do consumo de bebidas alcoólicas, este apresentou-se como fator de risco para o desenvolvimento de lesões de baixo e alto graus, com um aumento de 2,49 nas chances. No estudo em questão, 58,5\% das mulheres negaram o consumo de bebida, porém $41,4 \%$ informaram o consumo de álcool. Segundo pesquisa realizada por Nobre et al.
(2016), no registro do Instituto Nacional do Câncer (INCA), no período de 2005-2012, de 132.480 casos de câncer, 12,94\% consumiam bebida alcoólica, apresentando uma redução positiva com o passar dos anos para 7,37\%. Contudo, o hábito de consumir bebida alcoólica, independente de qual seja o seu teor alcoólico, tem relação direta com o desenvolvimento de neoplasias (MUNHOZ et al., 2016).

Em relação à frequência com que as mulheres costumavam ingerir carne vermelha, observou-se que $34,4 \%$ ingeriam de 3 a 4 dias na semana. Refrigerantes ou sucos artificiais destacaram-se como quase nunca ingeridos $(31,4 \%)$. O mesmo número de mulheres relatou consumir doces de 1 a 2 dias por semana e $40,0 \%$ afirmaram não trocar uma refeição por um lanche.

Em um estudo semelhante realizado em Teresina, PI, por Oliveira et al. (2014), apenas 5,9\% das mulheres estudadas costumavam ingerir carne vermelha. Já o consumo de refrigerantes foi relatado por $7,8 \%$ das mulheres em todos os dias da semana, e a ingesta de alimentos doces por $31,4 \%$ em pelo menos 1 vez por semana. A má alimentação tem-se apresentado como um importante influenciador no desenvolvimento de doenças crônicas não transmissíveis, paralelamente aos altos índices de obesidade e sobrepeso (OLIVEIRA et al., 2017).

Os hábitos considerados fatores de proteção também foram questionados, no qual $45 \%$ consumiam verduras e legumes todos os dias da semana e $50 \%$ mantinham o mesmo consumo com as frutas. Em estudo realizado em Campinas, SP, por Francisco et al. (2015), com 2.015 participantes, 34,4\% mantinham o hábito regular de consumir frutas e hortaliças e apenas $22,1 \%$ consumiam adequadamente os alimentos, atingindo o recomendado de cerca de 5 porções cotidianas em 5 dias da semana ou mais.

Dessa forma, observa-se que a amostra do estudo tem hábitos saudáveis quanto à alimentação. Porém, o consumo é insuficiente para manter uma nutrição equilibrada e adequada. Segundo Santos (2016), pode-se considerar que a ingesta de alimentos como frutas, verduras e legumes desencadeiam um fator protetivo contra certos tipos de cânceres, já que estes possuem componentes como os carotenoides, importantes redutores de danos causados ao Ácido desoxirribonucleico (DNA). 


\section{EM UNIDADE DE SAÚDE}

Em relação à prática de exercícios físicos, $54,3 \%$ realizavam algum tipo de atividade, destacando a caminhada com $31,4 \%$ como a mais praticada, enquanto $45,7 \%$ não realizavam nenhum tipo de exercício. Quando questionado como consideravam seu estado de saúde, a maior parte $(52,9 \%)$ avaliou-o como bom e $34,3 \%$ consideraram-no regular. Tal premissa corrobora o estudo de Oliveira et al. (2014), o qual observou que a maioria das participantes $(72,5 \%)$ também não praticavam nenhum tipo de exercício e, quando realizavam, a caminhada era a mais citada. No quesito estado de saúde, 53,0\% classificaram-se como regular e 39,2\% com um bom estado de saúde, fator que tem influência direta com a faixa etária, uma vez que, com o passar dos anos, aumentam as comorbidades e as preocupações com o estado de saúde.

Além disso, a prática de exercício físico apresentou-se como fator de risco para lesões intraepiteliais de baixo e alto grau $(2,506)$. Porém, é notável a importância da atividade física na promoção e manutenção de saúde, já que pode levar a alterações significativas no metabolismo, como, por exemplo, a melhora do desempenho cardiovascular, redução de adipócitos corpóreos, além de fortalecer a musculatura e melhorar a qualidade de vida de pacientes com doenças crônicas já instaladas (TRINDADE, 2018).

Quanto ao histórico ginecológico, 51,4\% não faziam uso de nenhum método contraceptivo, enquanto $35,7 \%$ utilizam o contraceptivo oral. Os dados se assemelham ao estudo de Dalla Libera et al. (2016a) realizado em Anápolis, Goiás, no qual cerca de $20 \%$ dos laudos citopatológicos estudados informavam o uso do anticoncepcional oral. Cabe ressaltar que o uso desse tipo de contraceptivo demonstrou-se como fator de risco no desenvolvimento de lesões celulares intraepiteliais, aumentando em 1,41 a chance de desenvolvê-las.

Segundo Almeida e Assis (2017), a iniciação do uso do contraceptivo hormonal oral antes de um completo amadurecimento do aparelho genital feminino tem-se demonstrado como um importante fator desencadeador de lesões precursoras do câncer de colo uterino. A sua utilização por tempo prolongado, a fim de se evitar gravidez indesejada, também tem se destacado, já que o mesmo pode aumentar a margem de transição e elevar os índices de eversão do tecido glandular, propiciando maior exposição ao HPV, assim como maiores danos ao tecido gerado pela presença da infecção (BARASUOL; SCHIMIDT, 2014).

A não utilização do método de barreira foi destacado por $30,0 \%$ das mulheres, dados inferiores ao estudo de Machado e Pires (2017) realizado no Estado de São Paulo, no qual 69,39\% das mulheres não utilizavam preservativos, assim como estudo de Nobre et al. (2014), que identificou o desuso da camisinha por $70,8 \%$ das mulheres. O uso de preservativo apresentou um aumento de 5,95 chances para desenvolver lesões de colo uterino. Porém, ressalta-se a possibilidade de viés de causalidade reversa, já que a maioria das mulheres que relatam não fazer uso do método de barreira são casadas e possuem parceiro fixo.

Portanto, o uso de anticoncepcionais hormonais apresenta-se como o principal fator envolvido na não utilização do método de barreira, porquanto a maioria das mulheres preocupam-se com a não geração de filhos e deixam de fazer uso do preservativo, o que as tornam suscetíveis a desenvolver IST e, tardiamente, lesões de alto grau (BEDIN; GASPARIN; PITILIN, 2017).

Considera-se, ainda, que o início precoce das atividades sexuais pode ser considerado um fator para desenvolver lesões precursoras do câncer de colo uterino, devido à exposição precoce às infecções sexualmente transmissíveis. No presente estudo, $57,1 \%$ das mulheres informaram iniciar a vida sexual entre 16 e 19 anos de idade, destacando a primeira menstruação entre 13 e 15 anos (54,3\%). Devido ao grande número de mulheres casadas, $68,6 \%$ das pesquisadas informaram apenas um parceiro sexual no último ano.

O estudo de Figueiredo et al. (2015) assemelhouse a este estudo, com $57 \%$ das mulheres com início da atividade sexual entre 15 e 19 anos, $89,2 \%$ com menarca entre 12 e 14 anos e, ainda, 67,7\% também informaram a prática sexual apenas com um parceiro. Logo, na pesquisa de Silva, Coelho e Athayde (2016), realizado em Montes Claros, MG, com afetadas pelo HPV, as idades de iniciação da relação sexual prevaleceram entre 16 e 20 anos $(61,6 \%)$, discordando no quesito multiplicidade de parceiros, tendo de 1 a 3 parceiros sexuais no último ano $(75,8 \%)$.

Aponta-se que o início precoce das relações sexuais pode interferir significativamente no desenvolvimento do câncer de colo uterino, visto que, na fase de puberdade, há um aumento na proliferação 
das células epiteliais localizadas na região ectocervical. Isso propicia uma maior susceptibilidade às infecções sexualmente transmissíveis e lesões precursoras do câncer (SILVA; COELHO; ATHAYDE, 2016).

No estudo ora apresentado, apenas $22,9 \%$ das mulheres informaram a infecção pelo HPV. Porém, $50,0 \%$ das pesquisadas apresentaram, em seus laudos citopatológicos, a infecção pelo vírus, observando-se um desconhecimento quanto à infecção ou mesmo ao diagnóstico da lesão por parte das pacientes.

Dentre as principais infecções genitais, o HPV destaca-se como um importante fator desencadeador do câncer. Cerca de 290 milhões de ocorrências de infecção pelo HPV são detectados em mulheres, sendo que $32 \%$ dessas são infectadas pelos subtipos com maior grau oncogênico. Além disso, uma vez portadora do vírus, a mulher pode apresentar uma regressão espontânea ou progredir para o câncer propriamente dito. Cabe ressaltar, aqui, que o fato de estar infectado pelo vírus não determinará o surgimento da neoplasia, já que se deve considerar a susceptibilidade de cada pessoa, os fatores de risco e o tempo de permanência do vírus no organismo (TAKITO; CAVALLI; GIEP, 2015).

A frequência da realização do exame citopatológico também foi questionado e $61,4 \%$ informaram realizar o exame anualmente e $15,7 \%$, raramente. Entretanto, quando questionado o motivo pela não realização do exame, 5,7\% informaram a falta de tempo e, $4,3 \%$ a vergonha. Tais afirmativas corroboram o estudo de Junior, Oliveira e Sá (2015), realizado em Diamantino, Mato Grosso, com uma amostra de 30 mulheres, no qual $46,6 \%$ das participantes relataram não realizar o exame por vergonha e $26,6 \%$ por ausência de tempo.

Outrossim, a vergonha é comumente relatada pelas mulheres, sendo considerada normal, já que há exposição do corpo durante o exame e muitas mulheres preocupam-se com o julgamento dos profissionais, o que, muitas vezes, acaba por adiar a realização do exame, retardando o diagnóstico (SILVA et al., 2018).

Neste estudo, 87,1\% das mulheres negaram ter tido casos de câncer de colo do útero na família. Porém, a variável em questão, quando cruzada com as lesões intraepiteliais de baixo e alto graus, demostrou ser um importante fator de risco, aumentando em 4,51 as chances de desenvolver as lesões. Estudo revela que a hereditariedade, acompanhada de outros fatores, principalmente no carcinoma altamente invasor, tem forte relação com o aparecimento da neoplasia, com maior evidência em parentes de primeiro grau (CARVALHO et al., 2015).

Segundo Pereira et al. (2017), o Brasil é considerado o país que mais consome agrotóxico no mundo, tendo expandido seu crescimento em até $190 \%$ nos últimos dez anos. A exposição a essa substância pode se dar não somente ao manuseio inadequado do produto, mas também através de muitos alimentos disponibilizados no comércio. Portanto, o uso indiscriminado dos agrotóxicos tem-se destacado como um importante desencadeador de neoplasias, dentre elas o câncer de colo uterino, alterando de forma significativa o DNA das células.

$\mathrm{Na}$ presente pesquisa, a exposição a esta substância foi informada por $28,6 \%$ das mulheres, sendo que $12,9 \%$ foram expostas durante a lavagem das roupas utilizadas para a aplicação do produto e $8,6 \%$ tiveram contato durante a própria aplicação. Em um estudo realizado através do Registro Hospitalar do Câncer incluindo os municípios da $10^{\circ}$ Regional de Saúde do Paraná, pode-se observar que, das neoplasias localizadas no aparelho reprodutor feminino, $14,44 \%$ destas tiveram relação com a exposição aos agrotóxicos (RUTHS, 2018).

Em relação às alterações encontradas nos laudos citopatológicos destas mulheres, $65,6 \%$ apresentaram lesões de baixo grau e 34,3\% lesões de alto grau, sendo que $1,4 \%$ apresentou, em laudo, o diagnóstico de câncer de colo do útero já instalado. Isso se contrapõe ao estudo de Figueiredo et al. (2015), que detectou $67,8 \%$ de lesões intraepiteliais de alto grau, incluindo casos do câncer.

Backes, Mezzari e Calil (2016) realizaram um estudo em Erexim, RS, com 62.280 laudos citopatológicos coletados pelo convênio do SUS, nos quais cerca 2.049 destes apresentaram algum tipo de alteração cervical, $47,49 \%$ foram diagnosticadas com células escamosas atípicas de significado indeterminado (ASC-US) e, após um curto período, 29,68\% tiveram uma evolução para lesões de alto grau.

Durante o período de coleta, observou-se a dificuldade apresentada pelas mulheres ao responder o questionário, principalmente nas questões voltadas às condições ginecológicas, às infecções às quais já estiveram expostas e ao diagnóstico atual da doença. Dessa forma, nota-se a ausência de conhecimento 


\section{EM UNIDADE DE SAÚDE}

dessas mulheres em relação às suas condições de saúde, uma vez que se pressupõe que, enquanto estão sendo acompanhadas periodicamente por um profissional, deixam de buscar informações e conhecimentos a respeito da patologia.

Enfim, como limitação do estudo, destaca-se a reorganização do serviço de saúde, no qual houve uma queda significativa na demanda da unidade, dificultando o alcance da amostra desejada.

\section{CONCLUSÃO}

Ao se efetivar o presente estudo, averiguou-se que os fatores de risco identificados na pesquisa foram o sobrepeso, consumo de bebidas alcoólicas, exposição ao agrotóxico e o histórico familiar de CCU. Sobre o perfil de mulheres com alteração citopatológica, o mesmo compõe-se de jovens, casadas, brancas, com baixas renda e escolarização. As mesmas fazem uso de método contraceptivo do tipo oral, apresentaram sexarca e menarca antes dos 16 anos e não faziam uso do método de barreira.

Alvitra-se que o referido estudo possibilitará o desenvolvimento de pesquisas futuras, as quais trarão maior conhecimento sobre os fatores desencadeadores de complicações de colo uterino destacados na região. Além disso, pode-se potencializar a educação em saúde, vinculada às informações fidedignas à população e melhor abordagem do assunto pelos profissionais de saúde. Tudo isso para minimizar os casos e alertar a população envolvida.

\section{REFERÊNCIAS}

ALMEIDA, A. F. et al. Methods for detection of cervical cancer among health professionals. Rev. Saúde Pública, Recife, v. 9, n. 1, p. 62-68, Jan., 2015.

ALMEIDA, A. P. F.; ASSIS, M. M. Efeitos colaterais e alterações fisiológicas relacionadas ao uso de contínuo de anticoncepcionais hormonais orais. Rev. eletrônica Atualiza Saúde, Salvador, v. 5, n. 5, p. 85-93, jan/jun., 2017.

BACKES, L. T. H.; MEZZARI, A.; CALIL, L. N. Población femenina asistió en citologico y cambios en sus prevalencia en cuello uterin. Extensio: Revista Eletrônica de Extensão, Florianópolis, v. 13, n. 22, p. 56-67, 2016.

BARASUOL, M. E. C.; SCHIMIDT, D. B. Cervical cancer and its risk factors: integrative review. Rev. Saúde e Desen., v. 6, n. 3, p. 139-153, jul/dez., 2014.
BARCELOS, M. R. B. et al. Qualidade do rastreamento do câncer de colo uterino no Brasil: avaliação externa do PMAQ. Rev. Saúde Pública, v. 51, p. 1-13, 2017.

BAZZO, K. et al. Lesões intra-epiteliais: relações com métodos contraceptivos orais, tabagismo e achados citológicos. In: IV Simpósio Científico FTSG de Graduação e Pós-Graduação, 2014, Bento Gonçalves. Simpósio Científico de Graduação e Pós-Graduação. Bento Gonçalves: FTSG, 2014. p. 1-14.

BEDIN, R.; GASARIN, V. A.; PITILIN, E. B. Factors associated to uterine-cervix changes in women assisted in a pole town in western Santa Catarina. Rev. Fund. Care. Online, Santa Catarina, v. 9, n. 1, p. 167-174, jan/mar., 2017.

BRASIL. Ministério da Saúde. Vigilância Alimentar e Nutricional - SISVAN. Orientações básicas para a coleta, o processamento e análise de dados e a informação em serviços de saúde. Série A. Normas e manuais técnicos. Brasília, 2004.

BRASIL. Ministério da Saúde. Portal Principal de Notícias da Saúde. Rio de Janeiro, 2017. Disponível em: < http:// portalms.saude.gov.br/component/content/article/804imc/40508-so-o-imc-nao-diz-como-voce-esta $>$.

CARVALHO, D. D. S. Perfil epidemiológico dos casos de câncer do colo do útero no Brasil e a sua associação com Chlamydia Trachomatis. 2016. 18f. TCC (Curso de Biomedicina) - Centro Universitário de Brasília, UniCEUB, Brasília, 2015.

DALlA LIBERA, L. S. et al. Human Papillomavirus infection evaluation in cytopathological exams. RBAC, Goiânia, v.48, n.2, p. 138-143, 2016a.

Cytological tests suggestive of infectionbyhuman papillomavirus. Estudos, Goiânia, v. 43, n. 1, p. 34-40, jan/ mar., $2016 \mathrm{~b}$.

DIAS, E. G. Socioeconomic profile and practice of cervical cancer prevention examination of uterus women of a health unit.Rev. Saúde e Desen., v. 7, n. 4, p. 133-146, jan/dez., 2015.

DUONG, M. N. et al.The fat and the bad: Mature adipocytes, key actors in tumor progression and resistance. Oncotarget, v. 8, n. 34, p. 57622-57641, 2017.

FIGUEIREDO, T. et al. Analysis of the profile of women with precancerous lesions of the cervix. Saúde em Revista, v. 15, n. 41, p. 3-13, set/dez., 2015.

FRANCISCO, P. M. S. B. et al. Sociodemographic inequalities in non communicable chronic disease risk and protection factors: telephone survey in Campinas, São Paulo, Brazil. Epidemiologia e Serviços de Saúde, Brasília, v. 24, p. 7-18, jan/mar., 2015. 
INSTITUTO NACIONAL DE CÂNCER (INCA). Incidência de Câncer no Brasil: Estimativa 2018, Rio de Janeiro, INCA, 2018. Disponível em: http://www.inca.gov. br /estimativa/2018/casos-taxas-brasil.asp.

JUNIOR, J. C. O.; DE OLIVEIRA, L. D.; DE SÁ, R. M. Factors of membership and not membership no women on examination pap smear. Rev. Eletrônica Gestão \& Saúde, v. 6, n. 1, p. 184-200, 2015.

LOBO, L. M. G. A.; ALMEIDA, M. M.; OLIVEIRA, F. B. M. Uterine column cancer, HPV and Papanicolaou experiment: a reflection on women's knowledge. Revista Ciência \& Saberes-Facema, v. 4, n. 1, p. 889-895, 2018.

MACHADO, L. S.; PIRES, M. C. Epidemiological profile of women with human papillomavirus who use the public health service. Rev. Baiana de Enferm., v. 31, n. 4, 2017.

MACHADO, H. S.; DE SOUZA, M. C.; GONÇALVES, S. J. C. Cervical cancer: Epidemiological and cytopathological analysis in the city of Vassouras-RJ. Revista PróUniverSUS, v. 8, n. 1, 2017.

MELLO, F. A.; GALLE, L. C.; PRADO, R. L. Prevention of cervical cancer in the conception of the female population of a city inland of São Paulo state.. Rev.Colloquium Vitae, v. 9, n. 2, p. 45-52, mai/ago., 2018.

MORAES, M. A. A. et al. Processo saúde doença das mulheres com câncer cérvico uterino nas redes de atenção. Rev. da Univ. Vale do Rio Verde, Três Corações, v. 14, n. 1, p. 355-365, jan/jul., 2016.

MOREIRA, T. R. et al. Perfil das mulheres usuárias do sus com lesões intraepiteliais em um município do oeste do paraná. Arq. Ciênc. Saúde UNIPAR, Umuarama, v. 21, n. 3, p. 181-186, set/dez. 2017.

MUNHOZ, M. P. et al. Effect of physical exercise and nutrition in cancer prevention. Rev. Odont. de Araçatuba, v. 37, n. 2, p. 09-16, 2016.

NASCIMENTO, G. W. C. et al. Cervical cancer screening coverage in the state of Minas Gerais, Brazil between 2000-2010: a study using data from the Cervical Cancer Information System (SISCOLO). Cad. Saúde Colet., Rio de Janeiro, v.23, n.3, p. 253-260, 2015.

NOBRE, C. M. et al. Profile of women with abnormal pap examination in southern country. Vittalle. Rev. de Ciênc. da Saúde. v. 26, n. 1, p. 29-36, 2014.

NOBRE, R. M. B. et al. Clinical and epidemiological profile of cancer occurred in the 2005-2012 period in the State of Paraná. Rev. de Epidem. Contr. de Inf., v. 1, n. 1, p. 125-135, 2016.

OLIVEIRA, A. C. et al. Risk and protection factors for women's health in the prevention of cervical cancer. Rev Rene. v. 15, n. 2, p. 240-248, mar/abr., 2014.
OLIVEIRA, V. S. et al. Analysis of the risk factors for chronic non-communicable disease: study with employees of a private institution. Saúde (Santa Maria), v. 43, n. 1, p. xx-xx, Jan/abr, 2017.

PEREIRA, V. G. M. et al. A relação entre o uso de agrotóxicos e o aumento do índice de câncer no Brasil. Rev. Gestão em foco, n. 9, p. 164-170, 2017.

PREFEITURA MUNICIPAL DE FRANCISCO BELTRÃO. Francisco Beltrão, 2018 Disponível em: $<\mathrm{http}: / /$ franciscobeltrao.pr.gov.br/secretarias/saude/departamentos /departamento-assistencia-em-saude/assistencia-medica/ $>$. Acesso em: 10 fev. de 2018.

RIBEIRO, J. F. et al. Sociodemographic and clinical profile of women with cancer of the cervix in a city of north. Rev. Eletr. Gest. e Saúde, v. 6, n. 2, p. 1367-1381, 2015.

ROCHA, S. M. M.; BAHIA, M. O.; ROCHA, C. A. M. Profiles of cervical screening tests performed in Casa da Mulher, Pará State, Brazil. Rev. Pan-Amaz Saúde, v. 7, n. 3, p. 51-55, 2016.

RUTHS, J. C. et al. Ocorrência de câncer na região oeste do Paraná: análise a partir da ocupação e exposição a agrotóxicos. 2018. 108f. Dissertação (Mestrado em Biociências e Saúde) - Universidade Estadual do Oeste do Paraná, Cascavel, 2018.

SANTOS, L. G. Consumo frequente de carne vermelha e processada e sua associação com o desenvolvimento de doenças crônicas não transmissíveis: uma análise a partir da saúde coletiva. 2015. 55f. TCC (Curso Bacharel de Saúde Coletiva) - Universidade de Brasília, Brasília, 2015.

SILVA, E. O.; COELHO, M. C. V.; ATHAYDE, L. A. Cytological changes associated with infection Human Papillomavirus in women attended in a hospital. Rev. Eletr. Gest. e Saúde, Ibituruna, v. 7, n. 1, p. 52-64, 2016.

SILVA, J. P. et al. Papanicolau exam: factors that influence the failure to perform the examination in women aged to 40-65 years. Arq. Ciênc. Saúde, v. 25, n. 2, p. 15-19, abr/ jun., 2018.

SILVA, R. T. et al. Contraceptivos orais e hábito tabagista são fatores de risco para lesões precursoras do câncer do colo uterino. In: III Congresso de Pesquisa e Extensão da FSG-RS, 2015, Caxias do Sul, FSG, 2015. p. 1275-1288.

TAKITO, D.; CAVALLI, L. O.; GRIEP, R. Hpv and cervical cancer of the uterus: epidemiological and cytopathological analysis in Cascavel - Paraná. Rev. Thêma et Scientia, v. 5, n. 2E, p. 14-20, jul/dez., 2015.

TIECKER, A. P. et al. Knowledge and preventive practices related to oncological Diseases of climateric women. Rev. Inter. de Est. em Saúde, Caçador, v. 7, n. 1, p. 165-175, 2018. 


\section{EM UNIDADE DE SAÚDE}

TRINDADE, A. C. A. C. Nível de atividade física e uso de serviços de saúde em mulheres sob tratamento adjuvante contra o câncer de mama com inibidores de aromatase. 2018. 69f. Dissertação (Mestrado em Programa de Pós-Graduação em Fisioterapia) - Faculdade de Ciências e Tecnologia - FCT/UNESP, Presidente Prudente, 2018. 